\title{
Analyse diachronique de l'efficience technique des systèmes de production bovin viande. Baisse de la productivité des facteurs variables sur 23 ans
}

Productivity and efficiency of Charolais suckler cattle farming systems: evolutions over 23 years

Patrick Veysset, Michel Lherm, Marielle Roulenc, Christophe Troquier et Didier Bébin

\section{(2) OpenEdition}

\section{Journals}

Édition électronique

URL : http://journals.openedition.org/economierurale/4776

DOI : $10.4000 /$ economierurale.4776

ISSN : 2105-2581

Éditeur

Société Française d'Économie Rurale (SFER)

Édition imprimée

Date de publication : 15 décembre 2015

Pagination : 149-169

ISSN : 0013-0559

Référence électronique

Patrick Veysset, Michel Lherm, Marielle Roulenc, Christophe Troquier et Didier Bébin, « Analyse diachronique de l'efficience technique des systèmes de production bovin viande. Baisse de la productivité des facteurs variables sur 23 ans », Économie rurale [En ligne], 349-350 | septembrenovembre 2015, mis en ligne le 15 décembre 2017, consulté le 22 avril 2019. URL : http:// journals.openedition.org/economierurale/4776; DOI : 10.4000/economierurale.4776 


\title{
Analyse diachronique de l'efficience technique des systèmes de production bovin viande Baisse de la productivité des facteurs variables sur 23 ans
}

\author{
Patrick VEYSSET, Michel LHERM, Marielle ROULENC, Christophe TROQUIER, Didier BÉBIN • INRA, \\ UMR1213 Herbivores, Saint-Genès-Champanelle ; Clermont Université, VetAgro Sup, UMR1213 \\ Herbivores, Clermont-Ferrand \\ veysset@clermont.inra.fr
}

\begin{abstract}
De 1990 à 2012, les exploitations d'élevage bovins viande françaises ont accru leur taille et leur productivité du travail de plus de $60 \%$ grâce, notamment, à une substitution travail/capital et à une simplification de certaines pratiques d'alimentation du troupeau, entre autres. L'efficience technique des systèmes de production, mesurée par le ratio volume de production hors aides/ volume des consommations intermédiaires a baissé de près de $20 \%$, alors que le revenu par travailleur s'est maintenu grâce aux aides et aux gains de productivité physique du travail. Cette efficience technique est positivement corrélée à l'autonomie alimentaire des exploitations, ellemême négativement corrélée à la taille des exploitations et des troupeaux. Alors que le volume de production par hectare de surface agricole utilisée a stagné, I'autonomie alimentaire par les fourrages (la valorisation de l'herbe) a perdu 6 points. Les 23 années de progrès génétique, technique, technologique et des connaissances ont accompagné l'augmentation continue de la taille des exploitations et de la productivité du travail, et ont donc juste permis de compenser la diminution d'efficience des systèmes de production.
\end{abstract}

MOTS-CLÉS : bovins viande, exploitation agricole, économie, efficience

\section{Productivity and efficiency of Charolais suckler cattle farming systems: evolutions over 23 years}

Over the past 23 years (1990-2012) French beef-cattle farms had increased their size and labor productivity by more than $60 \%$. These gains were possible thanks to a substitution work/capital and to a simplification of herd feeding practices. The efficiency of production systems is estimated by the ratio volume of agricultural production/volume of intermediate consumptions (inputs, services, capital consumption). This efficiency decreased by $20 \%$, while income per worker remained stable thanks to aids and subsidies and to the gains in labor productivity. The efficiency of the beefcattle farming systems is strongly positively correlated with the feed self-sufficiency, which is itself negatively correlated with the size of farms and herds. While the volume of agricultural products per hectare of agricultural area remained stable, the forage feed self-sufficient lost 6 points. 20 years of genetics, technical, technological and knowledge progress has thus helped offset lower efficiency strongly related to the continuous increase in the size of the farms (JEL: Q120).

KEYWORDS: Beef cattle, farming system, economics, efficiency 
D e tous les secteurs de l'économie française, c'est l'agriculture qui a connu l'accroissement le plus rapide de la productivité du travail au cours des cinquante dernières années (Guihard, Lesdos, 2007). La production en volume de la branche a été multipliée par 2,2 entre 1955 et 2010, grâce à la spécialisation des exploitations, à l'utilisation croissante des intrants et à la mobilisation d'un capital toujours plus important (matériel et bâtiments). Sur cette même période, la population active agricole est passée de $31 \%$ à 3,4 \% de l'emploi total. Ce mouvement continu d'agrandissement et de spécialisation des exploitations est confirmé lors de chaque recensement agricole (Desrier, 2011 ; Giroux, 2011), et touche l'ensemble des productions, y compris la filière viande bovine (Institut de l'Élevage, 2011). Pourtant, depuis la fin des années 1990, et malgré une productivité du travail toujours en augmentation au sein des exploitations agricoles professionnelles, on observe un difficile maintien du revenu et un essoufflement de la productivité des autres facteurs (consommations intermédiaires, capital et terre [Butault, 2006]). Différentes études montrent qu'en exploitations d'élevage herbivores, l'accroissement de la productivité physique du travail ne va pas forcément de pair avec les principaux indicateurs économiques (Charroin et al., 2012).

L'agrandissement des exploitations d'élevage, et donc des troupeaux, à maind'œuvre constante, met la question du travail au cœur des préoccupations des éleveurs (Madelrieux, Dedieu, 2008). Pour résoudre ces problèmes de travail, les éleveurs tendent très souvent à simplifier leurs pratiques. Pour les exploitations bovins viande, ces simplifications de pratiques touchent principalement la gestion de l'alimentation et de la reproduction (Hostiou, Fagon, 2012). La gestion du travail sur l'exploitation n'est pas sans conséquences sur les performances animales (Martel et al., 2008) et la simplification peut s'accompagner d'une sous-valorisation des aliments de la ration (Agabriel et al., 2012), d'où une plus forte consommation de fourrages conservés et surtout de concentrés pour un même niveau de production.

Cette dépendance accrue aux intrants peut fragiliser, économiquement parlant, les systèmes de production dans un contexte d'augmentation du prix de l'énergie. Cette fragilisation potentielle incite les éleveurs à rechercher des systèmes de production plus autonomes, plus durables, notamment via la recherche d'autonomie alimentaire à l'échelle de leur exploitation (Havet et al., 2014 ; Coquil et al., 2014 ; Ryschawy et al., 2013). Les systèmes de polyculture-élevage sont alors cités comme pouvant répondre à cette recherche d'autonomie et de durabilité (Bell, Moore, 2012 ; Ryschawy et al., 2012 ; Salton et al., 2014). L'autonomie alimentaire est souvent associée à des systèmes d'élevage économes en intrants (Lherm, Benoit, 2003), et ainsi économiquement performants (RipollBosch et al., 2014) et créateurs de valeur ajoutée (Garambois, Devienne, 2012), y compris dans des exploitations herbagères (Garambois, 2011).

Entre agrandissement des structures, simplification des pratiques et recherche de systèmes économiquement durables via l'autonomie, nous pouvons nous interroger sur les conséquences de ces pratiques et sur l'efficience globale du système de production. L'objectif de cet article est d'observer si ces évolutions structurelles se sont accompagnées de gains de productivité des facteurs variables. Nous analyserons les évolutions sur 23 ans (1990-2012) des résultats productifs, des consommations intermédiaires, de capital, de la valeur ajoutée et du revenu des exploitations professionnelles françaises bovins viande. Pour fournir plus de détails sur les pratiques et résultats techniques, ainsi que sur la variabilité intra-annuelle, 
nous analyserons conjointement les performances d'un groupe constant de 43 exploitations du bassin charolais.

\section{Contexte et méthodes}

\section{Réseaux d'exploitations}

Exploitations bovins viande, réseau d'information comptable agricole (RICA)

Le RICA est un réseau harmonisé au niveau européen fournissant des informations statistiques sur la comptabilité, le revenu et l'économie des exploitations agricoles (Rouquette, Baschet, 2010). Il est représentatif de l'ensemble des exploitations agricoles françaises métropolitaines dites « professionnelles », c'est-à-dire dont le produit brut standard est supérieur à 25000 euros et employant au moins 0,75 unité de travail annuel. Les exploitations du RICA sont classées par région selon une typologie fondée sur leur orientation technico-économique (OTEX) et dimension économique (Cdex) respective. Le RICA est constitué d'un échantillon d'exploitations enquêtées annuellement, elles-mêmes pondérées par un coefficient d'extrapolation afin de représenter l'ensemble des exploitations professionnelles de l'OTEX considérée (Agreste, 2014). En 2010, le champ couvert par le RICA correspond à 96,8\% de la production agricole métropolitaine brute standard, $92 \%$ de la Surface agricole utile (SAU) et $62,2 \%$ de l'ensemble des exploitations agricoles.

Les exploitations professionnelles spécialisées bovins viande sont classées dans l'OTEX46. Ainsi, dans l'OTEX46, 566 exploitations enquêtées en 1990 en représentent 33 362. En 2012, les 33098 exploitations professionnelles bovins viande sont représentées par 719 exploitations.

\section{Le réseau INRA d'exploitations bovines allaitantes Charolais}

Afin de comprendre les déterminants des évolutions des exploitations d'élevage bovin allaitant, de connaître et d'analyser les potentialités techniques et économiques des systèmes de production, une équipe d'économie de l'INRA de Clermont-Theix a mis en place, depuis les années 1970, un réseau d'observations d'exploitations charolaises sur le long terme (Carrère, Liénard, 1976).

Chacune des fermes du réseau est enquêtée annuellement. Les données collectées concernent la main-d'œuvre, la structure, les surfaces et l'assolement, le troupeau, les consommations intermédiaires, les ventes, les aides et subventions, les investissements et les emprunts. L'ensemble des variables technico-économiques calculées à partir de ces enquêtes s'inscrit dans une vision de gestion technico-économique de l'exploitation et non comptable et fiscale. Sur les 71 exploitations suivies en 2013, nous avons constitué un groupe échantillon constant de 43 exploitations conventionnelles, toutes situées dans le bassin charolais, de 1990 à 2012. Afin de comparer les résultats annuels en termes d'autonomie et d'efficience, nous avons exclu de notre échantillon les exploitations ayant constitué (ou abandonné) un atelier secondaire (hors-sol) en cours de trajectoire.

\section{Principales évolutions structurelles observées entre 1990 et 2012}

Les principales évolutions structurelles des exploitations bovins viande sont marquées par (tableau 1) :

- une très forte augmentation de la taille, surface et troupeau, avec un léger recul de la main-d'œuvre totale disponible ;

- une forte augmentation de la productivité du travail (surface et nombre d'animaux par unité de main-d'œuvre, + 56 à $+68 \%$ )

- un maintien des systèmes herbagers, avec une extensification ;

- une forte capitalisation par travailleur (+ 31 à $+57 \%$ en euros constants). 
Les 43 exploitations charolaises du réseau INRA étaient en 1990, et restent en 2012, de taille significativement supérieure à celle du RICA, mais avec plus de main-d'œuvre. Cependant, les évolutions constatées au sein des deux réseaux sont comparables et parallèles, avec quelques nuances. Les exploitations du RICA étant plus petites, elles se sont simplement un peu plus agrandies en proportion : surface totale de l'exploitation exprimée en hectares de Surface agricole utile (ha/SAU) $+64 \%$ vs $+53 \%$ pour les Charolais ; taille des troupeaux exprimée en nombre d'Unités gros bovin (UGB) $+53 \% v s+47 \%$ pour les Charolais. Dans les deux échantillons, l'accroissement de la surface totale a été régulier et continu sur la période, ceci peut être mis en relation avec une attribution non plafonnée de l'ensemble des aides de la Politique agricole commune (PAC) ramené à l'hectare, qu'elles aient été couplées avant 2006 ou découplées après (Veysset et al., 2014a).

On observe une différence dans les dynamiques d'accroissement de la taille des troupeaux. Dans l'échantillon RICA, cet accroissement est continu et régulier jusqu'en 2007 (de 73 à 109 UGB sur 17 ans), puis s'infléchit sur les 5 dernières années (de 109 à 112 UGB) avec une légère baisse sur les deux dernières années (1 UGB de moins entre 2011 et 2012). Dans le réseau Charolais INRA, nous observons une croissance de la taille des troupeaux régulière de 1990 à 2001 (de 123 à 171 UGB), une quasi-stagnation de 2001 à 2007 (de 171 à 173 UGB), une reprise de l'agrandissement entre 2007 et 2010 (de 173 à 186 UGB) et enfin une légère baisse sur les 2 dernières années (186 UGB en 2010, 182 UGB en 2012). Ces inflexions dans la dynamique de croissance des troupeaux peuvent être liées à des changements dans l'attribution des aides PAC couplées à l'animal, et notamment du complément extensif (Veysset et al., 2014a) : seuil d'attribution peu contraignant (mode de calcul du chargement d'éligibilité) pour les exploitations herbagères du bassin charolais jusqu'en 2001, d'où une croissance de la taille des troupeaux sans renoncement à cette aide ; de 2001 à 2006, réévaluation du montant unitaire de ce complément extensif avec durcissement des conditions d'attribution (nouveau mode de calcul du chargement d'éligibilité), l'incitation économique a été suffisante, les éleveurs ont limité la taille de leur troupeau pour ne pas dépasser ce seuil de chargement ; après 2006, découplage de ce complément extensif, d'où la possibilité d'une reprise de croissance de la taille des troupeaux sans conséquence financière sur cette aide, alors que les éleveurs engagés dans la Prime à l'herbe agro-environnementale (PHAE) devaient toujours contenir leur chargement.

Les exploitations du réseau Charolais sont restées très spécialisées, elles n'ont pas eu de tendance à la " céréalisation » et ont même accru de 3 points leur spécialisation bovine (part du produit bovin hors aides dans le produit brut d'exploitation hors aide), alors que le rapport entre produit bovin et autres produits est resté stable dans le RICA Un mouvement de désintensification de la surface fourragère est observé dans les deux réseaux, les chargements y sont de même niveau.

Malgré cette forte augmentation de la productivité du travail, le revenu par travailleur est resté relativement stable dans les deux réseaux, avec de fortes variations interannuelles (figure 1). Les évolutions des deux échantillons sont parallèles, la différence de revenu entre RICA et Charolais INRA est due à la différence de taille et de productivité du travail.

Les évolutions structurelles, productives, techniques et économiques des exploitations du réseau Charolais INRA sur la période, ainsi que leurs déterminants, ont été décrites en détail et analysées par Veysset et al., 2014a. 
Tableau 1. Principales caractéristiques structurelles 1990 et 2012 des exploitations bovins viande du RICA (OTEX46) et du groupe constant de 43 exploitations du réseau Charolais INRA

\begin{tabular}{|l|l|c|c|c|}
\cline { 3 - 5 } \multicolumn{2}{l|}{} & 1990 & 2012 & Évolution \% \\
\hline \multirow{2}{*}{ UTH $^{*}$ totaux } & RICA OTEX46 & 1,41 & 1,38 & -2 \\
\hline & INRA Charolais & 2,09 & 2,00 & -4 \\
\hline \multirow{2}{*}{ SAU* ha / UTH) } & RICA OTEX46 & $64(45)$ & $105(76)$ & $+64(+68)$ \\
\cline { 2 - 5 } & INRA Charolais & $122(58)$ & $186(93)$ & $+53(+62)$ \\
\hline \multirow{2}{*}{ UGB ${ }^{*}$ totaux (/ UTH) } & RICA OTEX46 & $73(52)$ & $112(81)$ & $+53(+56)$ \\
\cline { 2 - 5 } & INRA Charolais & $123(59)$ & $182(92)$ & $+47(+57)$ \\
\hline \multirow{2}{*}{ SFP*\% SAU } & RICA OTEX46 & 84 & 85 & +1 \\
\hline \multirow{2}{*}{ Chargement/UGB ha SFP } & INRA Charolais & 82 & 83 & +1 \\
\hline \multirow{2}{*}{ Spécialisation bovine } & RICA OTEX46 & 1,30 & 1,20 & -4 \\
\cline { 2 - 5 } & INRA Charolais & 1,29 & 1,22 & -5 \\
\hline \multirow{2}{*}{ Capital total k $€ /$ UTH $^{* * *}$} & RICA OTEX46 & 85 & 84 & $\approx$ \\
\cline { 2 - 5 } & INRA Charolais & 83 & 86 & +3 \\
\hline & RICA OTEX46 & 177 & 232 & +31 \\
\hline & INRA Charolais & 182 & 285 & +57 \\
\hline
\end{tabular}

Notes : * $U T H=$ unité travailleur humain ; $S A U=$ surface agricole utile; UGB = unité gros bovins; $S F P=$ surface fourragère principale ; ${ }^{* *}$ produit brut bovin hors aides/produit brut d'exploitation hors aides ; ${ }^{* * *}$ capital total hors foncier : euros constants 2012 (déflateur : indice des prix à la consommation INSEE)

Source : les auteurs.

Figure 1. Évolution 1990-2012 du revenu (résultat courant, RC) des exploitations bovins viande en euros constants par travailleur (UTH) non salarié

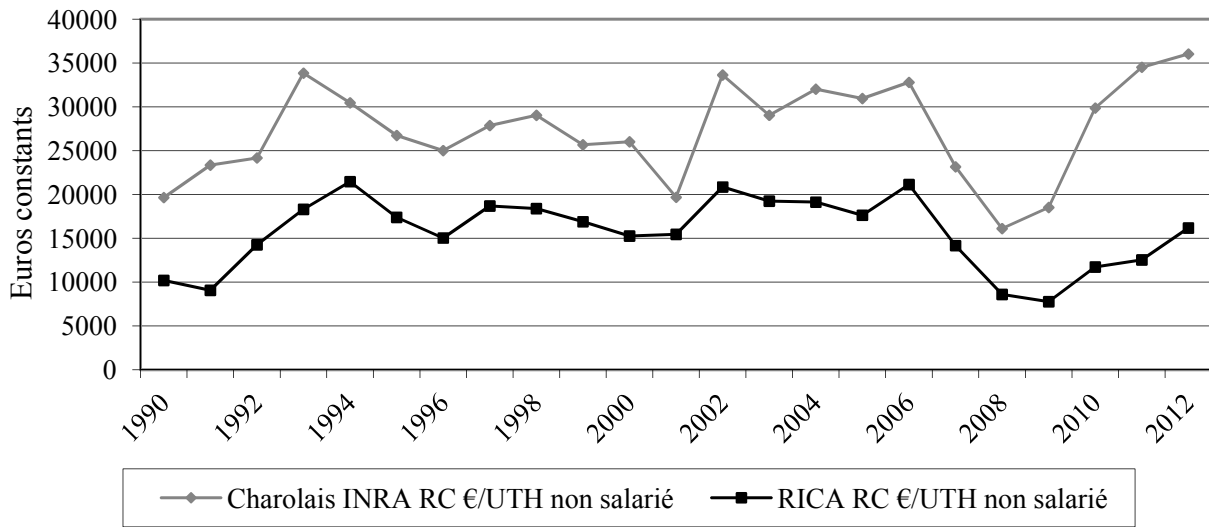

Source : les auteurs. 


\section{Productivité et efficience technique du système}

L'efficience d'une entreprise est sa capacité à obtenir de bonnes performances avec une quantité de facteurs de production donnée. Les performances peuvent être qualifiées de bonnes en les comparant à une référence (une autre entreprise, ou une autre année pour la même entreprise). L'efficience calculée d'une entreprise peut donc être mise en relation avec la productivité des facteurs de cette entreprise (Latruffe, 2010). On peut définir la productivité comme étant la capacité des facteurs de production à produire des biens et services. On peut la mesurer sous la forme d'un indicateur de productivité partielle, en mettant une production en rapport avec un intrant. Mais cette approche ne prend pas en compte l'ensemble des productions et des intrants de l'entreprise. L'agrégation de toutes les productions avec l'agrégation de tous les intrants permet de calculer la richesse créée par l'entreprise (ou valeur ajoutée) qui peut être utilisée dans un cadre dynamique d'analyse de l'évolution de la productivité (Latruffe, 2010) ; une entreprise ayant amélioré sa productivité sur une période donnée sera qualifiée de plus efficiente. Ce gain de productivité (ou d'efficience) peut être dû à une amélioration de l'efficacité (meilleure valorisation des mêmes intrants), à des économies d'échelle (une augmentation de la taille de l'entreprise entraîne une moindre augmentation des intrants par rapport à celle de la production), ou au progrès technologique.

\section{Concept de valeur ajoutée}

La valeur ajoutée est la différence entre les biens et services produits par une entreprise (hors aides et subventions) au cours d'un exercice, et les consommations externes (ou intermédiaires) : consommations de biens, services acquis auprès d'un tiers pour obtenir cette production. La valeur ajoutée nette est calculée en déduisant de la valeur ajoutée (brute) les amortissements, c'est-à-dire la consommation annuelle de capital fixe. La valeur ajoutée nette exprime la création de richesse du système de production. Après transfert de richesse opérée par la collectivité (ajout des aides et subventions), la valeur ajoutée nette est répartie entre rémunération du foncier (fermage), rémunération de la main-d'œuvre extérieure, cotisations sociales, intérêts du capital emprunté, taxes sur le foncier et les produits, pour aboutir au résultat courant avant impôts (ou revenu agricole).

La valeur ajoutée par actif ou par ha de surface permet de comparer l'efficacité économique des systèmes de production hors subventions (Cochet, Devienne, 2006).

\section{Productivité des facteurs variables et efficience technique du système de production}

L'efficience technique du système de production sera évaluée au travers de la productivité des facteurs variables utilisés afin d'observer si les exploitations bovins viande ont su (ou pu) créer de la richesse au cours des années. Selon le concept de valeur ajoutée, le produit est la Production agricole hors aides (Prod) et les ressources utilisées (ou facteurs variables) sont les Consommations intermédiaires (CI) et la Consommation de capital fixe (CKf). L'efficience technique du système (Eff) sera donc représentée par l'évolution du ratio :

$$
\operatorname{Prod} /(\mathrm{CI}+\mathrm{CKf})
$$

L'intensité d'utilisation des intrants (c'est-à-dire $\mathrm{CI} / \mathrm{Prod}$ ), en valeur économique, peut être utilisée pour calculer un score de durabilité économique des exploitations (Lebacq et al., 2015). Cette efficience est dépendante de l'évolution des volumes (effet volume) et des prix (effet prix) respectifs des produits et des facteurs variables de production. Ce que nous cherchons à appréhender ici est la productivité 
«physique » de ces CI et CKf, c'est-à-dire le rapport des volumes de produits et des moyens de production, avec la difficulté d'agréger le volume des différentes productions (viande, céréale, etc.) et des différents facteurs (CI : engrais, aliments, fuel, services, etc.). En corrigeant l'effet prix, les évolutions annuelles des valeurs économiques observées des produits, les consommations intermédiaires et les amortissements (matériel, bâtiment et installations), nous capterons l'effet volume. Pour ce, il faut distinguer pour chaque poste les volumes et les prix unitaires (Butault et al., 1995). Cette décomposition est relativement complexe, par contre l'évolution annuelle des volumes produits ou consommés de chaque poste peut être approchée si l'on connaît l'indice des prix respectif de chacun des postes relativement à une année de référence.

\section{Indices IPPAP et IPAMPA}

L'Indice des prix des produits agricoles à la production (IPPAP) vise à mesurer l'évolution des prix de vente des produits par les agriculteurs. Chaque produit agricole est l'objet d'observations successives de sa valeur, et l'INSEE en fournit l'indice (Thibert, Berger, 2009). La base 100 des derniers indices fournis est l'année 2010. Les valeurs annuelles de chaque production recensée par le RICA dans l'OTEX46 (céréales, cultures industrielles, autres produits végétaux, bovins, ovins, porcs, volailles, produits animaux, autres produits) ont été corrigées de leur IPPAP respectif lorsque ceux-ci sont clairement identifiés (céréales, gros bovins, ovins, porcins, volailles, autres animaux). Les cultures industrielles et autres produits végétaux, qui ne représentent que 2 à $5 \%$ de la production annuelle, ont été corrigées de l'IPPAP « oléagineux », le colza étant souvent présent dans les assolements, en rotation avec le blé, dans les exploitations polyculture-élevage bovin viande. Les « autres produits », représentant également
2 à $5 \%$ de la production annuelle, ont été corrigés de l'indice général hors fruits et légumes. Pour le réseau Charolais INRA, nous avons également pondéré les produits annuels de leur IPPAP respectif, mais nous l'avons fait pour chaque éleveur, et non sur la moyenne annuelle de l'échantillon. De plus, pour cet échantillon, nous disposons du détail des produits vendus et avons ainsi pu décomposer le produit bovin de chaque éleveur entre bovins maigres et bovins de boucherie, pour les pondérer des IPPAP bovins maigres et IPPAP bovins de boucherie.

L'INSEE suit également les prix unitaires de biens et services utilisés par les exploitants dans le cadre de leur activité agricole pour calculer l'Indice des prix d'achat des moyens de produits agricoles (IPAMPA) (Berger et al., 2000). L'IPAMPA recouvre neuf postes de consommations intermédiaires et investissements (semences, engrais et amendements, vétérinaires, produits de protection des cultures, aliment du bétail, petit outillage, énergie, biens d'équipement, conseil et frais généraux). Ces neuf postes permettent de couvrir plus de $70 \%$ des charges réelles d'une exploitation d'élevage. La base 100 de l'IPAMPA est également l'année 2010. Tout comme pour les produits, les valeurs annuelles moyennes de chaque poste de charge de l'échantillon RICA, et chaque poste de charge de chaque exploitation de notre réseau Charolais ont été corrigées respectivement de leur IPAMPA annuel. Les postes de charges n'ayant pas d'IPAMPA sont agrégés avec des postes dont l'IPAMPA est connu (par exemple, les frais d'élevage tels que l'insémination artificielle ou le contrôle de performance sont agrégés avec les frais vétérinaires).

\section{Expression et variabilité des résultats}

Les évolutions structurelles du secteur de l'élevage bovin font que le travail et 
sa productivité occupent une place centrale dans la gestion des systèmes d'élevage (Hostiou, Dedieu, 2011). L'élevage d'herbivores a également la caractéristique d'être fortement lié au sol pour l'alimentation des animaux. Les volumes produits ainsi que ceux des facteurs variables consommés seront exprimés par hectare de surface agricole utile (ha/SAU) et par Unité de travail humain (UTH). L'évolution de la productivité du foncier et du travail sera donc captée par celle du volume de richesse créée (valeur ajoutée nette), et de ses composantes, par ha/SAU et par UTH. Nous commenterons ensuite l'évolution annuelle du ratio d'efficience technique.

Les résultats présentés sont les valeurs moyennes annuelles respectives de l'ensemble des exploitations constituant les deux échantillons. Dans le réseau Charolais-INRA, nous disposons des valeurs de l'ensemble des variables structurelles, techniques et économiques pour chacune des 43 exploitations chaque année. Une Analyse en composantes principales (ACP) avec les principales variables structurelles et techniques en variables actives (41 variables), nous permettra de résumer la variabilité des résultats interexploitations. Vu les dynamiques d'évolution de la productivité du foncier et du travail (cf. infra), nous pouvons distinguer trois sous-périodes au cours des 23 années étudiées : 1990-2001, 2001-2007 et 2007-2012. Nous avons réalisé trois ACP regroupant 43 exploitations * 12 années $=516$ observations pour la période 1990 $2001,43 * 7=301$ observations pour 2001-2017 et $43 * 6=258$ observations pour 2007-2012. En projetant sur les axes issus des ACP les variables de production, consommation intermédiaire et capital fixe, valeur ajoutée et efficience, nous pourrons observer à quels éléments de variabilité sont corrélées ses variables, et l'évolution entre sous-périodes de ces corrélations.

\section{Résultats}

\section{Valeur ajoutée et ses composantes Productivité du foncier et du travail}

Exploitations bovins viande RICA, OTEX46

Le volume de biens agricoles produits par ha de SAU par les exploitations bovins viande françaises n'a globalement pas évolué au cours des 23 dernières années (figure 2). Le volume de production par ha a même tendance à baisser depuis 2007 du fait d'une moindre augmentation de la taille des troupeaux relativement à celle de la surface agricole. Cette stagnation de la productivité du foncier est liée à la désintensification de la surface fourragère (moins d'animaux produits par ha de surface fourragère principale, SFP), elle-même accompagnée d'une moindre consommation d'engrais (-54\% en volume), de semences $(-23 \%)$ et de produits phytosanitaires (-17\%). Mais ces charges de cultures sont les seuls postes à avoir baissé en volume sur la période étudiée. Les quantités utilisées par ha de SAU de tous les autres postes de consommations intermédiaires ont augmenté, dont les principaux : aliments du bétail $+21 \%$, produits vétérinaires $+14 \%$, carburant $+29 \%$, entretien et réparation du matériel $+33 \%$, eaux, gaz, gestion et autres services +17\%. Globalement, le volume de l'ensemble des consommations intermédiaires par ha de SAU a augmenté de 0,64 \% par an. Malgré l'agrandissement des exploitations, les consommations annuelles de capital fixe par hectare de SAU augmentent également, avec un taux d'augmentation de $1,25 \%$ par an. Le poste matériel en est le principal responsable, il représente $68 \%$ des consommations annuelles de capital fixe. Les dynamiques d'évolution des volumes de production agricole, de consommations intermédiaires et de capital fixe par ha SAU ont le même profil : une légère baisse de 1990 à 1995, signe d'un attentisme face à la nouvelle réforme de la PAC 
de 1992, pour revenir à la valeur de 1990 en 2001 ; puis une hausse des consommations jusqu'en 2007 avec une stagnation de la production, signe d'une confiance liée au niveau des aides perçues et à la stabilité de l'ensemble prix de la viande vendue + aides ; et enfin une légère baisse depuis 2007 certainement liée à la lente érosion de l'ensemble prix + aides (Veysset et al., 2014a).

La valeur ajoutée nette produite par ha de SAU chute avec un taux moyen annuel d'évolution de $-1,08 \%$ (figure 3). Elle est négative, c'est-à-dire que la production agricole est inférieure aux CI et CKf engagées. De 1990 à 2001, les volumes consommés de facteurs variables augmentent légèrement plus vite que ceux produits par ha SAU d'où une tendance à la baisse de la valeur ajoutée nette par ha SAU, de 2001 à 2007 face à une stagnation de la production et à une augmentation constante des consommations de facteurs variables, la valeur ajoutée nette par ha SAU chute fortement, et enfin, depuis 2007, le volume de richesse créée par ha de SAU stagne. Du fait de la forte augmentation de la taille des troupeaux à main-d'œuvre constante le volume de production agricole par travailleur (UTH) augmente de $67 \%$ entre 1990 et 2012 (figure 4). Mais les volumes utilisés de consommations intermédiaires et de capital fixe par UTH augmentent respectivement sur la même période de $72 \%$ et $70 \%$. Tout comme pour la productivité du foncier, l'augmentation de la production par travailleur s'est réalisée grâce à une forte augmentation d'utilisation d'aliments du bétail, de carburants et de mécanisation (investissement et entretien). Alors que la production par travailleur augmente fortement, le volume de richesse créée (valeur ajoutée nette) par UTH perd $113 \%$ (figure 3). Les dynamiques d'évolution de la productivité du travail et du foncier sont parallèles (figure 3).

Figure 2. Évolution des volumes de productions, consommations intermédiaires et consommations de capital fixe par ha de SAU (€2010 déflatés de IPPAP et IPAMPA)

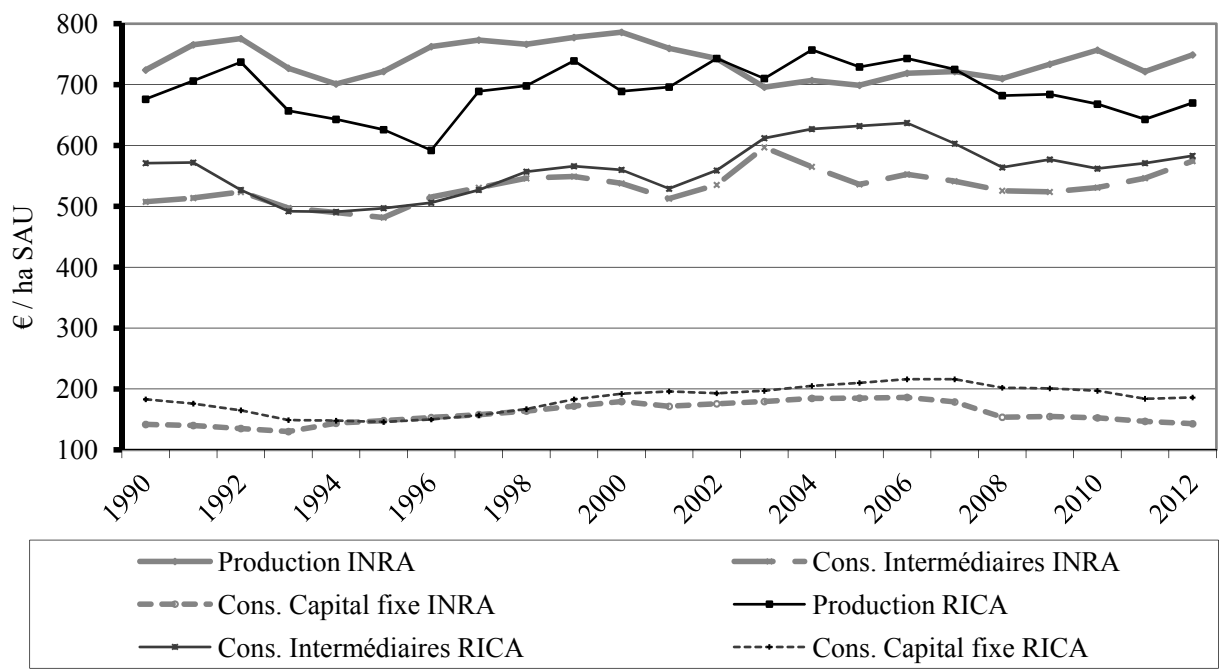

Source : les auteurs. 
Figure 3. Évolution de la valeur ajoutée nette par ha de SAU et par UTH ( $€ 2010$ déflatés de IPPAP et IPAMPA)

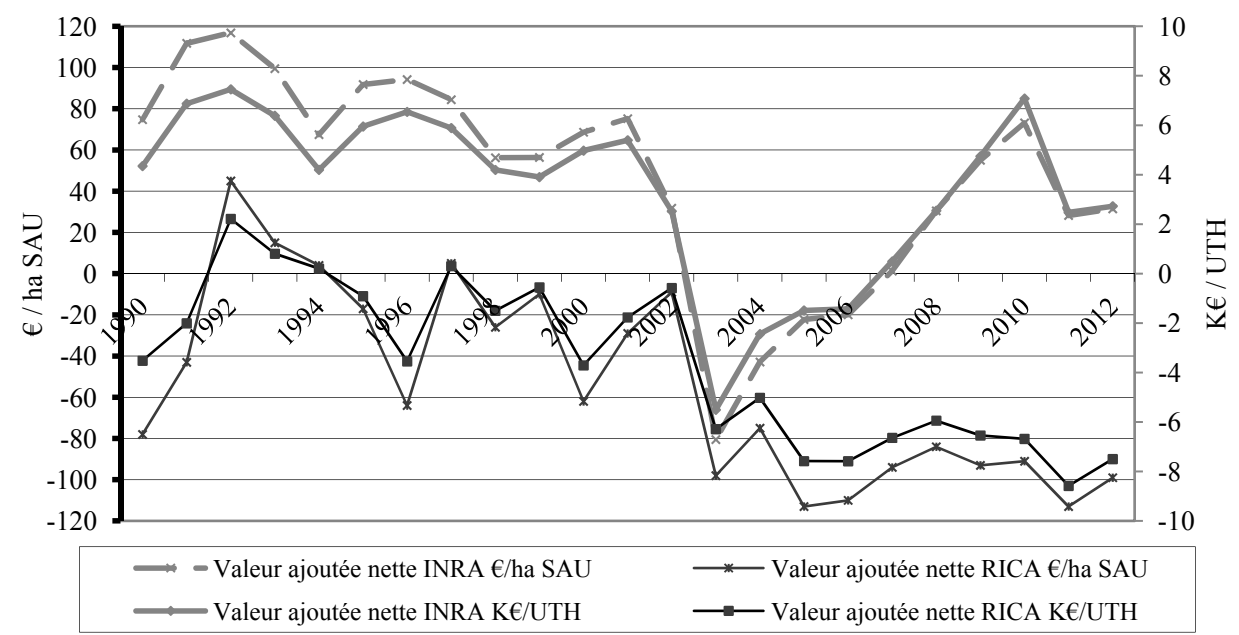

Source : les auteurs.

Figure 4. Évolution des volumes de productions, consommations intermédiaires et consommations de capital fixe par UTH ( $€ 2010$ déflatés de IPPAP et IPAMPA)

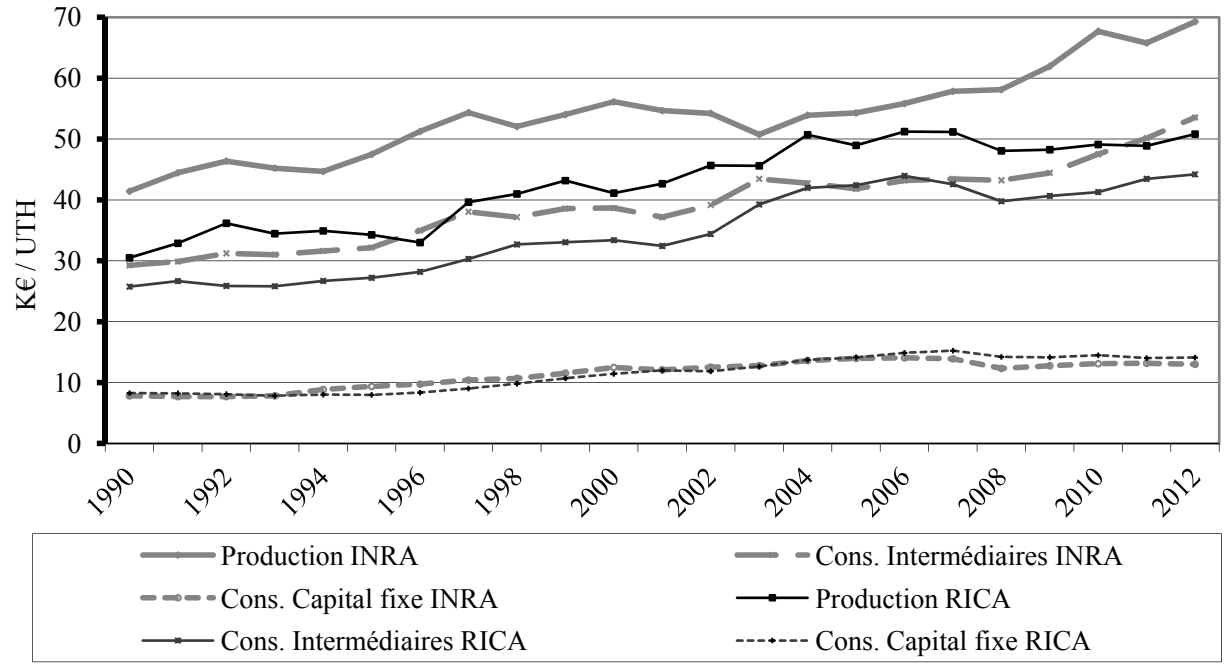

Source : les auteurs. 
Réseau Charolais INRA, analyse technique

Les évolutions annuelles de la production, des consommations intermédiaires et du capital fixe par hectare de SAU des élevages du réseau Charolais INRA sont parallèles et comparables à celles observées sur les exploitations de l'univers RICA jusqu'en 2007 (figure 2). À partir de 2007 les courbes de production par ha SAU divergent avec une augmentation des volumes produits observée dans le réseau Charolais INRA. Alors que les volumes utilisés de facteurs variables par UTH sont parallèles entre les deux réseaux, le profil des volumes produits par travailleur est différent (figure 4). Dans le réseau RICA, la production par UTH augmente de 1990 à 2004, puis stagne. Dans le réseau Charolais INRA, elle augmente jusqu'en 2000, stagne de 2000 à 2005, puis augmente assez fortement depuis 2005 .

Cette divergence depuis 2005-2007 des volumes produits par ha SAU et par UTH s'explique par la différence observée de l'évolution de la taille des troupeaux entre les deux réseaux. Le volume de richesse créée par ha de SAU et par UTH évolue puis baisse, parallèlement entre les deux réseaux jusqu'en 2001. Après une forte chute en 2003, la valeur ajoutée nette produite par le réseau Charolais INRA réaugmente sans atteindre le niveau du début des années 1990, alors que celle du RICA stagne (figure 3).

\section{Production agricole hors aides}

Les parts relatives des produits animaux et végétaux dans le produit global hors aides sont restées relativement stables sur la période d'étude. Le produit bovin représente $83 \%$ du produit total hors aides, les céréales $9 \%$, les oléagineux $4 \%$, les $4 \%$ restant se partagent entre ovins, autres animaux et divers.

La productivité numérique (nombre de veaux sevrés pour 100 femelles mises à la reproduction) perd 1,9 point en 23 ans (86,4 \% en 2013 vs 88,3\% en 1990). Cette baisse de productivité numérique est liée à une baisse du taux de gestation (de $94 \%$ à $91 \%$ ) et à une légère augmentation de la mortalité des veaux : de 7,5\% à 9,5\%, tendances observées dans les grands troupeaux (Veysset et al., 2004). Du fait de la demande de la filière, et principalement de la forte et constante demande des ateliers d'engraissement italiens en broutards jeunes et lourds, la part des animaux engraissés baisse : en 2012 seulement $26 \%$ des mâles vendus sont engraissés sur la ferme contre $42 \%$ en 1990. Mais l'augmentation du gabarit des animaux (le poids de carcasse des vaches de réforme gagne $50 \mathrm{~kg}$, soit $+13 \%$ ) permet de compenser cette baisse de productivité numérique et d'engraissement, la production de viande vive par UGB (productivité pondérale) passe de 295 à $327 \mathrm{~kg}$ entre 1990 et 2012, soit un gain de $10 \%$.

Plus d'UGB par travailleur, et des UGB plus lourds, la production de viande vive par travailleur gagne $75 \%$ en 23 ans, de 17 à 30 tonnes/UTH. Ce qui explique l'augmentation du volume de la production agricole par travailleur.

Le chargement s'est maintenu autour de $1,30 \mathrm{UGB} / \mathrm{ha}$ de surface fourragère principale (SFP) jusqu'en 2001. Le renforcement du complément extensif par l'Agenda 2000 (réforme de la Politique agricole commune en 2000) a été très incitatif, le chargement moyen est passé sous les 1,25 UGB/ha SFP à partir de 2002, et s'est maintenu à ce niveau depuis (le découplage du complément extensif en 2006 n'a pas encouragé une réintensification). Cette baisse du chargement vient quasiment annuler les gains de productivité pondérale, la production de viande vive par ha de SFP n'augmente que de $15 \mathrm{~kg}$ en 23 ans (+5\%).

Sur l'ensemble de la période, le rendement des céréales est resté stable autour de 47 quintaux de grains/ha (alors que dans 
l'échantillon RICA le rendement céréales passe de 50 à 60 qx/ha).

La stagnation des productivités respectives des surfaces fourragères et des surfaces en cultures explique la non-évolution en 23 ans du volume de production de biens agricoles par ha de SAU.

\section{Consommations intermédiaires et de capital fixe}

La désintensification de la surface fourragère n'a pas empêché une recherche d'amélioration de la quantité et de la qualité des fourrages récoltés : la part de la surface en herbe fauchée annuellement passe de $38 \%$ en 1990 à $47 \%$ en 2012, et la part de la surface en herbe ensilée et/ou enrubannée sur cette surface fauchée passe de $7 \%$ à $21 \%$. La part de maïs fourrage dans la SFP, toujours très limitée, baisse de $6 \%$ à $3 \%$. Malgré une baisse de l'engraissement des animaux et une plus grande disponibilité en fourrages conservés sous forme d'ensilage ou enrubannage, théoriquement de meilleure valeur alimentaire que le foin, la quantité de concentrés distribués par $\mathrm{kg}$ de viande vive produite (kgvv) augmente très fortement : $+33 \%$ en 24 ans $(1,63 \mathrm{~kg}$ concentrés/kgvv en 1990 vs 2,17 en 2012). Notons que l'utilisation, pour produire un $\mathrm{kg}$ de viande vive, de concentrés produits sur l'exploitation (autofournis) et achetés a augmenté dans les mêmes proportions. La conséquence est une baisse de 6 points en 23 ans de l'autonomie alimentaire énergétique (UF) par les fourrages (part des besoins annuels UF du troupeau couverts par les UF des ressources fourragères de l'exploitation : $88 \%$ en 1990 vs $82 \%$ en 2012) et de 3 points de l'autonomie alimentaire UF globale (part des besoins annuels UF du troupeau couverts par les UF de l'ensemble des ressources de l'exploitation : $94 \%$ en 1990 vs $91 \%$ en 2012). La forte utilisation de concentrés autofournis permet de limiter la baisse d'autonomie alimentaire globale, par contre elle s'oppose à une meilleure valorisation des fourrages.
En contrepartie de la stagnation de la productivité du foncier, nous observons une moindre consommation d'engrais par ha de SAU : la fertilisation azotée minérale moyenne passe de $50 \mathrm{~kg} \mathrm{~N} / \mathrm{ha}$ à $41 \mathrm{~kg} \mathrm{~N} /$ ha, soit $-18 \%$. La consommation de l'ensemble des charges de cultures (engrais, semences, traitement) baisse de $35 \%$. Mais, tous des autres postes de consommations intermédiaires augmentent en volume par ha de SAU : les aliments du bétail achetés (+38\%), l'énergie (+55\%), le petit outillage et entretien du matériel (+65\%), les frais généraux $(+12 \%)$, les charges de vétérinaires et autres frais d'élevage $(+12 \%)$. Globalement le volume de l'ensemble des consommations intermédiaires par ha de SAU augmente de $13 \%$ (figure 3).

Après une forte augmentation jusqu'en 2006, la consommation de capital fixe par hectare de SAU a eu tendance à baisser depuis. L'augmentation des consommations de capital entre 1990 et 2006 peut être due aux forts investissements en bâtiments (modernisation, aménagement et/ ou construction) liés aux Plans de maîtrise de pollution d'origine agricole (PMPOA de 1994 à 2007). Cette modernisation des bâtiments d'élevage a également entraîné des investissements en nouveaux matériels tels que des pailleuses, dessileuses, mélangeuses, et autres matériels de distribution de fourrages et concentrés. Avec l'apparition de l'enrubannage au début des années 1990 et le développement des presses balles rondes à haute densité, l'ensemble de la chaîne récolte/stockage/distribution de fourrages s'est modernisée au cours des années 1990 (Liénard et al., 1998). Globalement, le volume de consommation de capital fixe par ha de SAU augmente de $1,5 \%$ (figure 3 ).

Face à l'augmentation de $67 \%$ du volume production agricole par travailleur, le volume de consommations intermédiaires par travailleur augmente de $83 \%$, et celui de capital fixe de $66 \%$ (figure 4 ). 
Le volume de richesse produit par ha de SAU et par UTH chute respectivement de $58 \%$ et $37 \%$ (figure 3 ).

\section{Réseau Charolais INRA, variabilité des résultats}

Sur la période 1990-2001, la variabilité de l'échantillon peut être résumée autour de deux axes, la constitution des autres axes étant relativement diffuse. Sur 20012007 et 2007-2012, on identifie clairement quatre axes résumant $68 \%$ de la variabilité de l'échantillon. Les deux premiers axes, constitués des mêmes variables pour les trois sous-périodes, expliquent $47 \%$ de la variabilité totale.

Les variables contribuant au premier axe sont les variables de taille de l'atelier bovin, et donc de l'exploitation vu le taux de spécialisation : nombre de travailleurs totaux, ha SAU, nombre d'UGB, kg totaux de viande vive produite, ha de SFP, ha de cultures intra-consommés, part du maïs fourrage dans la SFP. À ces variables s'ajoutent des variables d'alimentation des animaux : les quantités de concentrés distribuées aux animaux et utilisées par $\mathrm{kg}$ de viande produits sont corrélées positivement à cet axe, l'autonomie alimentaire y est corrélée négativement. Cet axe discrimine donc les grandes exploitations bovines utilisant beaucoup de concentrés intra-consommés et/ou achetés pour produire une forte quantité de viande.

Le second axe est constitué par les variables exprimant la spécialisation des exploitations. En coordonnées positives nous trouvons la spécialisation bovine, la part de SFP dans la SAU ; en coordonnées négatives se trouvent la surface en culture, le rendement en céréales et la quantité d'azote minérale par ha SAU.

Le volume de production agricole par ha/SAU et par UTH, ainsi que le volume de consommations intermédiaires par ha/ SAU et par UTH, sont fortement corrélés positivement à l'axe taille. Les grandes exploitations sont donc les plus productives par ha SAU et par UTH, mais avec force utilisation d'intrants. Les consommations de capital fixe par ha SAU sont indépendantes de la taille (alors que, d'après le concept d'économie d'échelle, on pourrait s'attendre à une corrélation négative), exprimées par UTH, elles sont corrélées positivement à la taille. Globalement, sur les trois sous-périodes, le créé par ha de SAU et par UTH n'est pas lié à la taille : le volume de facteurs variables consommés en plus est juste compensé par le volume de production, il n'y a pas d'économie d'échelle.

Sur la période 1990-2001, la spécialisation en production bovine (second axe) n'influe pas sur le volume de valeur ajoutée par ha/SAU et par UTH. Par contre sur 2001-2007, et surtout 2007-2012, nous observons une corrélation négative entre la spécialisation bovine et la richesse créée (ou une corrélation positive entre production de céréales et richesse créée). La désintensification fourragère induit une stagnation (voire une légère baisse) de la production de viande bovine par ha SAU alors que la modernisation des bâtiments d'élevage et de la chaîne de récolte entraîne une augmentation de la consommation de capital fixe par ha SAU, d'où un léger effet dépressif de la spécialisation bovine sur le volume de richesse créée par ha de SAU. Un hectare de céréale étant moins gourmand en maind'œuvre qu'un hectare de SFP pour la production bovine, la corrélation entre volume de production agricole par UTH et spécialisation bovine est donc négative. Mais cette corrélation était annulée, sur 1990-2001, par le fait que beaucoup d'intrants étaient utilisés sur les cultures. À partir de 2001, nous observons une baisse d'utilisation d'engrais minéraux et de produits phytosanitaires sur les cultures, alors que l'utilisation d'aliments concentrés achetés continue d'augmenter par kg de viande produit; la richesse créée par UTH est alors positivement corrélée à la part de cultures de 
vente dans l'exploitation, et négativement à la spécialisation bovine.

Le troisième axe, exprimant $13 \%$ de la variabilité de l'échantillon sur les périodes 2001-2007 et 2007-2012 est constitué des variables exprimant le pourcentage d'animaux engraissés sur la ferme, mais également l'intensification de la production de viande par ha et par UGB. À l'opposé, sur ce même axe, se trouve la taille de l'exploitation par travailleur. Cet axe discrimine donc les engraisseurs intensifs à surface limitée par travailleur. Enfin, le quatrième axe discrimine les exploitations selon leur niveau d'autonomie alimentaire et donc d'aliment concentré acheté. Il apparaît que le volume de richesse créée, par ha de SAU et par UTH, est assez fortement lié à l'autonomie alimentaire, celle-ci permettant de limiter les consommations intermédiaires (par ha et par UTH).

\section{Efficience du système de production et revenu par travailleur}

Un volume de production agricole par ha/ SAU stable avec un plus fort recours aux consommations intermédiaires et de capitaux par ha SAU : l'efficience technique des systèmes de production s'est donc dégradée sur les 23 dernières années, de façon parallèle sur les deux réseaux (figure 5). La dégradation annuelle de la productivité des facteurs variables de production est de $-0,7 \%$ par an pour les exploitations bovins viande du RICA et de $-0,8 \%$ par an pour celles du réseau Charolais INRA.

Au début des années 1990, ces exploitations du réseau Charolais INRA apportaient $20 \%$ de valeur aux quantités de consommations intermédiaires et de capitaux utilisées, d'où création de richesse (efficience $=1,2$ ). À partir des années 2000 , il y a transfert de valeur, mais pas création de richesse (efficience $=1$ ).

Sur les deux réseaux, nous observons un point bas en 2003, année de forte canicule et de sécheresse. Ce point bas est également observé pour les valeurs ajoutées nettes (figure 3). En 2003, la production de viande a peu été affectée par la sécheresse, les éleveurs ayant acheté des fourrages et concentrés pour maintenir le poids des animaux (Veysset et al., 2007), d'où un pic de consommations intermédiaires. Les rendements de céréales ont été affectés par cette sécheresse : -20 à $-25 \%$ pour le réseau

Figure 5. Efficience technique des systèmes de production : évolution de la productivité des facteurs variables

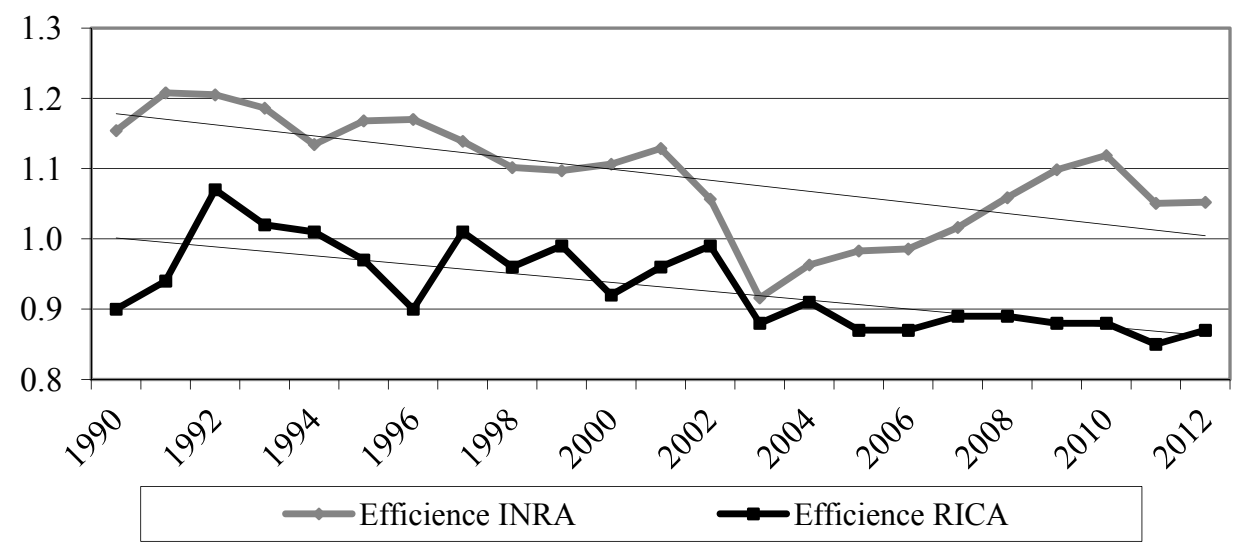

Source : les auteurs. 
Charolais, $-10 \%$ sur les exploitations du RICA, d'où une baisse de productivité des facteurs variables et de valeur ajoutée plus marquée en 2003 sur le réseau Charolais. Après 2003, la productivité des facteurs variables des exploitations du RICA stagne et ne retrouve pas son niveau d'avant 2003 du fait de la stagnation de la taille des troupeaux. Par contre, après la forte baisse, les exploitations du réseau Charolais ont tendance à regagner en efficience technique grâce à l'augmentation du volume de production agricole (liée à l'augmentation de la taille des troupeaux), mais à un niveau inférieur aux années précédant 2003. Ce creux beaucoup plus marqué en 2003 pour les exploitations du réseau Charolais peut s'expliquer par leur taille supérieure à celle des exploitations du RICA. En effet, les mauvaises années, le fort niveau des charges de structure incompressibles des grandes exploitations amplifie la chute de leur revenu (Veysset et al., 2005).

La projection de ce ratio de productivité sur les axes de nos ACP montre un léger effet dépressif de la taille (corrélation négative avec le premier axe) pour la sous-période 2007-2012, alors que la corrélation avec cet axe est nulle sur les deux autres sous-périodes. Sur 2001-2007 et 2007-2012, la spécialisation en production bovine (deuxième axe) influe négativement sur l'efficience technique des systèmes du fait de la forte augmentation de l'utilisation d'aliments concentrés pour la production de viande. L'intensification de la production de viande avec engraissement des animaux avait un effet positif sur l'efficience technique des systèmes sur 2001-2007, effet nul sur 2007-2012. Enfin, le quatrième axe, c'est-à-dire l'autonomie alimentaire, est celui auquel est le plus fortement lié, positivement, cette efficience. La productivité des facteurs variables est, depuis 2001, corrélée positivement à l'autonomie alimentaire, ellemême corrélée négativement à la taille et au volume de production de viande de l'exploitation.

Cette productivité des facteurs variables est un déterminant positif du revenu par travailleur (figure 6).

Figure 6. Relation entre productivité des facteurs variables et revenu par travailleur. 43 exploitations sur 23 ans $=989$ observations. Revenu en euros constant 2012, déflateur indice des prix à la consommation INSEE

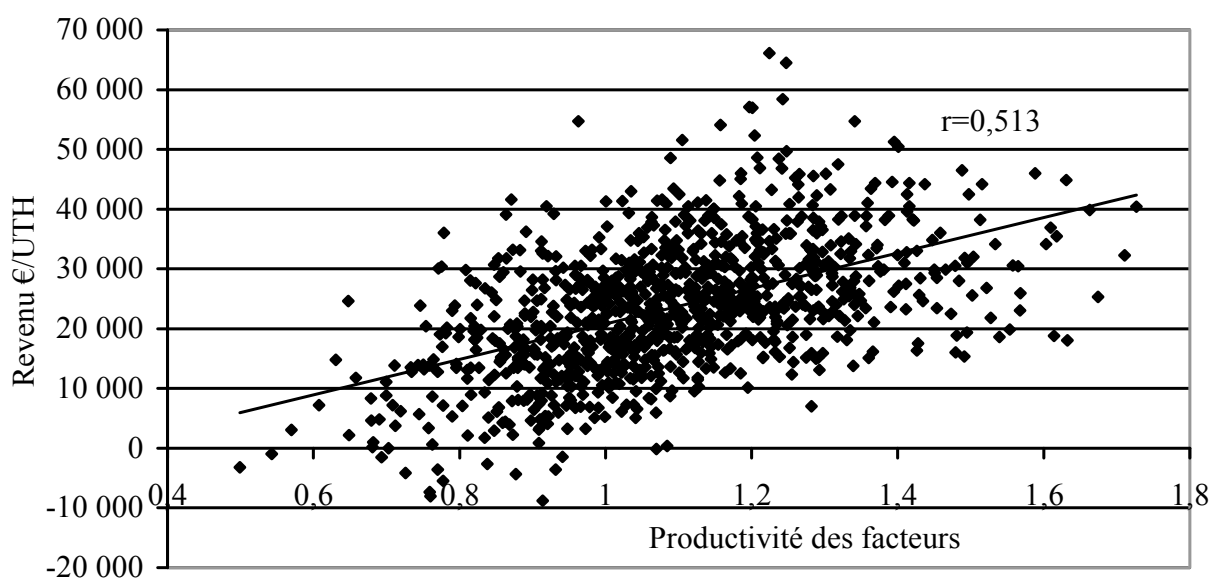

Source : les auteurs. 


\section{Discussion}

\section{Efficience comparée d'autres productions agricoles}

Nous avons appliqué la même méthodologie (utilisation des IPPAP et IPAMPA respectifs pour calculer les volumes de production, consommations intermédiaires et de capital fixe par ha de SAU) aux résultats annuels des orientations technicoéconomiques bovin lait (RICA OTEX45) et céréales, oléagineux, protéagineux (RICA OTEX15). La productivité des facteurs variables de ces deux spéculations a progressé, de 1990 à 2012, à un rythme de $+0,34 \% / a n$ et $+1,08 \%$ par an respectivement pour les bovins lait et grandes cultures.

La progression est plus significative pour les grandes cultures. Elle est liée à une progression du volume de production (+0,86 \% par an) couplée à une stagnation des consommations intermédiaires et à une régression de la consommation de capital fixe par ha/SAU (-0,8 \% par an). Ces exploitations de grandes cultures ont consommé près de $30 \%$ d'engrais de moins par ha/SAU (premier poste de charge), par contre les postes travaux par tiers et entretien du matériel ont augmenté respectivement de $73 \%$ et $25 \%$, le poste carburant n'augmente que de $8 \%$.

Grâce à une meilleure productivité des vaches laitières (lait produit par vache et par an : 4750 1. en 1990 vs 6610 l. en 2013, soit $+40 \%$ ), accompagnée d'une baisse limitée du chargement $(-7 \%)$, le volume de production par ha/SAU gagne $17 \%$ en 23 ans, soit un taux de $+0,72 \% /$ an. Comme pour les autres productions, la consommation d'engrais par ha a considérablement baissé $(-55 \%)$. Les autres postes de consommations intermédiaires par ha augmentent (aliments du bétail $+21 \%$, produits vétérinaires $+54 \%$, travaux par tiers $+21 \%$, entretien du matériel $+31 \%$, carburants $+38 \%$, autres fournitures et services $+30 \%$ ). Globalement le volume de consommations intermédiaires utilisées par ha augmente de $9 \%$. La consommation de capital fixe par ha augmente de $19 \%$, soit un taux de $+1,18 \%$ par an. Contrairement aux bovins viande, le volume de production en bovin lait a augmenté plus rapidement que celui des facteurs variables, d'où un gain de productivité de ces facteurs.

Il semble que pour les productions bovines, lait et viande, l'agrandissement et la modernisation des fermes ont entraîné une forte consommation de mécanisation (travaux par tiers, entretien matériel, amortissement matériel) et de carburant. L'ensemble des tâches des exploitations de grandes cultures était déjà mécanisé en 1990. Ces exploitations ont utilisé les progrès technologiques et l'amélioration des connaissances pour améliorer l'efficience de la mécanisation et des consommations intermédiaires.

\section{2. Économie d'échelle et économies de gammes ?}

Au cours des 23 années observées, les aides et la forte augmentation de la productivité physique du travail ont juste permis de maintenir le revenu par travailleur des exploitations bovins viande. Ces gains de productivité ont été "redistribués » (Boussemart et al., 2012) vers l'aval de la filière (baisse des prix des produits agricoles) et vers l'amont (agrofournitures et machinisme). L'agrandissement n'a donc pas été source d'économies d'échelle. La consommation de capital fixe, en volume, ne se dilue pas avec le nombre d'ha, au contraire elle augmente du fait des nouveaux besoins en mécanisation que génère l'augmentation de la taille (surface et troupeaux). Les relations entre taille des exploitations agricoles et efficience des systèmes de production ne font pas toujours consensus. Alors que divers auteurs soulignent les gains d'efficience avec l'augmentation de la taille des exploitations (Morrison Paul 
et al., 2004 ; Mosheim, Knox Lowell, 2009), d'autres montrent que la relation n'est pas forcément linéaire, et que l'efficience peut décroître au-delà d'une certaine taille (Helfand, Levine, 2004 ; Jaforullah, Whiteman, 1999). La productivité d'un facteur de production peut être améliorée par son échelle d'utilisation (économie d'échelle), mais l'augmentation de la taille de l'exploitation peut induire l'accès à de nouvelles technologies, techniques et pratiques qui peuvent améliorer la productivité (économie de taille). Cette distinction entre économie d'échelle et économie de taille (Hallam, 1991) permet de supposer que la compétitivité des plus petites fermes peut être augmentée par l'amélioration de l'efficience technique (adoption de meilleures technologies et/ou pratiques) plutôt que par l'accroissement de leur taille (Sheng et al., 2014 ; Singbo, Larue, 2014). Dans les exploitations bovins viande étudiées, il semblerait que l'augmentation de la taille génère l'adoption de matériel et pratiques (chaîne de récolte et de distribution de fourrage entièrement mécanisée) n'ayant pas d'effet sur la productivité (pas d'économie de taille), ce qui inciterait les éleveurs à s'agrandir pour amortir ces nouvelles technologies (économie d'échelle).

De même, la diversification polyculture-élevage n'entraîne pas forcément des économies de gamme (Perrot et al., 2013 ; Veysset et al., 2014b). Les exploitations disposant de plus de surfaces en céréales utilisent plus de concentrés par $\mathrm{kg}$ de viande vive produits (corrélation négative entre autonomie alimentaire par les fourrages et surface en cultures).

On assiste donc à une dégradation continue, depuis au moins 20 ans, de la productivité des facteurs variables et de la richesse créée par ces exploitations en parallèle de l'agrandissement continu et de la part de plus en plus importante des aides dans l'économie de l'exploitation. Cette baisse d'efficience technique se cumule avec un ciseau des prix défavorable (les prix d'achat des moyens de production augmentent plus vite que celui des produits agricoles), le revenu hors aides par travailleur s'effondre littéralement (RICA OTEX46 : 1248 €/UTH en 1990 vs - 17594 $€ / U T H$ en 2013. Réseau Charolais INRA : $6640 € / \mathrm{UTH}$ en 1990 vs -19 940 €/UTH en 2013, € constants 2013, déflateur indice des prix à la consommation INSEE).

Hors aides, le revenu par travailleur est donc largement négatif depuis 1995. La taille a longtemps été un fort moteur du maintien du revenu des éleveurs, du fait, dans un premier temps, de l'augmentation du volume de production, puis, à partir du milieu des années 1990, des aides supplémentaires qu'apportait un agrandissement (aides couplées à l'animal et/ou à l'hectare, Veysset et al., 2005). Mais cette corrélation taille-revenu par travailleur s'est continuellement amenuisée. La corrélation avec la taille du troupeau devient même proche de zéro ces cinq dernières années du fait de la dégradation des performances zootechniques des grands troupeaux, associée à de plus fortes consommations de concentrés pour simplifier le travail.

\section{Pratiques alimentaires, autonomie alimentaire et efficience}

L'autonomie alimentaire est un point clé de l'efficience technique des systèmes de production bovin viande et de la valeur ajoutée créée par ces systèmes. Mais cette autonomie alimentaire est négativement corrélée à la taille des exploitations et au volume de viande bovine produit.

Un des objectifs de la réforme de la Politique agricole commune en 1992, dite Mac Sharry, a été de favoriser, inciter, l'incorporation de céréales communautaires dans l'alimentation des animaux en substitution de sous-produits céréaliers importés (corn-gluten). Cette incitation s'est réalisée via une forte chute du prix des céréales (-50\% en euros constants entre 1992 et 
2005). Les éleveurs ont ainsi pu augmenter la taille des troupeaux, simplifier les pratiques alimentaires en distribuant plus d'aliments concentrés (faciles à stocker et à distribuer, et à valeur alimentaire connue et stable) sans que la valeur de la charge alimentaire n' augmente fortement.

Cette forte utilisation de céréales dans l'alimentation des bovins s'est accompagnée d'une évolution du mode de récolte de l'herbe avec le développement de l'enrubannage. Ce mode de récolte et de conservation des fourrages influence de manière très significative les consommations de carburants qu'elles soient ramenées à l'UGB ou à l'hectare de SFP (Devun et al., 2014) et peut, en partie, expliquer la très forte hausse de consommation d'énergie par ha à l'échelle de l'exploitation. Ces nouvelles pratiques alimentaires allant vers une amélioration de la concentration énergétique des rations, tout en consommant plus d'énergie fossile, ne sont pas cohérentes avec une stagnation de la production de viande à l'hectare et s'opposent à la valorisation des fourrages de l'exploitation.

\section{*}

La productivité des facteurs variables de production des exploitations bovins viande a, au cours des 23 dernières années, baissé. L'évolution de l'efficience technique de ces systèmes de production peut être mise en relation avec celle de la taille des exploitations et notamment des troupeaux. La dynamique de la taille des troupeaux est elle-même en partie influencée par le mode d'attribution des aides et subventions (à la tête, à la surface, conditionnée ou non à un seuil de chargement). Nous pouvons ainsi distinguer trois sous-périodes au cours des 23 années étudiées : i) 1990-2001 avec la mise en place des mesures de la réforme de la PAC de 1992 et une lente érosion du volume de richesse créée par ha et par UTH, ainsi que de la productivité des facteurs variables, la course aux aides est engagée au détriment de la gestion technico-économique ; ii) 2001-2007 avec le renforcement des mesures de 1992 par la réforme de 2000, et une baisse plus accentuée de l'efficience des systèmes liée à une augmentation du volume de consommations intermédiaires et à une baisse de l'autonomie alimentaire, les tendances observées sur 1990-2001 se renforcent ; iii) 20072012 avec le découplage partiel des aides, une stabilisation de consommation des facteurs variables pour un volume de production stable (RICA) ou en augmentation (Charolais INRA) d'où une stagnation, voire une amélioration, de l'efficience des systèmes et de la richesse créée, mais à un niveau inférieur aux valeurs observées sur 1990-2001.

L'agrandissement continu des exploitations, la recherche de toujours plus de productivité physique du travail, la course aux aides et surtout leur optimisation, ont induit une plus forte consommation de ressources externes (intrants et capital) au détriment d'une meilleure valorisation des ressources internes (potentiel génétique des animaux et végétaux) sans augmenter la productivité des surfaces utilisées. La recherche d'autonomie alimentaire est une question des éleveurs très actuelle. Cette demande de références, cette prise de conscience de l'importance de la gestion technico-économique des ateliers de production, est pressante sous l'effet de l'augmentation du prix des céréales et donc des aliments concentrés, depuis 2007. Cette quête de l'autonomie alimentaire dépend probablement moins de progrès technologiques ou de nouvelles connaissances, que de l'amélioration des pratiques alimentaires et de la gestion des troupeaux et surtout de la valorisation de l'existant.

Dans une filière de production bovin viande où le foncier et le travail sont deux facteurs limitants, le fait que le volume de richesse créée par ha de SAU et par UTH 
ait baissé en 23 ans devrait amener à nous interroger collectivement sur le modèle de développement de cette production et sur l'utilisation des fruits de plus de 20 années de progrès génétiques, technologiques, techniques et des connaissances. On peut penser que tous les progrès ont convergé vers l'augmentation « sans limites » de la productivité physique du travail de l'éleveur, sans se soucier de l'efficience globale de ce travail. Une analyse des comptes de surplus devrait nous permettre de décrire comment se répartissent les gains de productivité.

\section{RÉFÉRENCES BIBLIOGRAPHIQUES}

Agabriel J., Farrie J.-P., Pottier E., Note P., Pomies D. (2012). Conséquences zootechniques de simplifications de pratiques : exemple de la distribution des aliments et de la traite des vaches. INRA Productions Animales, vol. 25, $\mathrm{n}^{\circ}$ 2, pp. 141-158.

Agreste (2014). Rica France : tableaux Disar. Réseau d'information comptable agricole, $16 \mathrm{p}$.

Bell L. W., Moore A. D. (2012). Integrated crop-livestock systems in Australian agriculture: trends, drivers and implications. Agricultural Systems, n 111, pp. 1-12.

Berger J., Lemarquis D., Pochet M., Pous B. (2000). L'IPAMPA (indice des prix d'achat des moyens de production agricole) - Méthodologie. INSEE Méthodes, ${ }^{\circ}$ 94, 157 p.

Boussemart J.-P., Butault J.-P., Ojo O. (2012). Generation and distribution of productivity gains in French agriculture. Who are the winners and the losers over the last fifty Years? Bulletin USAMV, Hoticulture, vol. $69, \mathrm{n}^{\circ} 2$, pp. 55-67.

Butault J.-P. (2006). La baisse des revenus et l'essoufflement de la productivité dans l'agriculture française depuis 1998. INRA Sciences Sociales, $\mathrm{n}^{\circ}$ 2, $8 \mathrm{p}$.

Butault J.-P., Delame N., Rousselle J. M. (1995). Formation et répartition des gains de productivité dans l'agriculture française, analyse par produit. Cahiers d'Économie et de Sociologie Rurales, $\mathrm{n}^{\circ} 33$, pp. 55-72.

Carrère G., Liénard G. (1976). En élevage bovin charolais, quelles innovations techniques ? Évolutions technico-économiques réalisées par un groupe d'éleveurs de bovins charolais de la Nièvre (1971-1975). Bulletin Technique C.R.Z.V. Theix, INRA, $\mathrm{n}^{\circ} 26$, pp. 35-53.

Charoin T., Veysset P., Devienne S., Fromont J. L., Palazon R., Ferrand M. (2012). Productivité du travail et économie en élevage herbivores : définition des concepts, analyse et enjeux. INRA Productions Animales, $\mathrm{n}^{\circ}$ 25, pp. 193-210.

Cochet H., Devienne S. (2006). Fonctionnement et performances économiques des systèmes de production agricole : une démarche à l'échelle régionale. Cahiers Agricultures, vol. $15, \mathrm{n}^{\circ} 6$, pp. 578-583.

Coquil X., Beguin P., Dedieu B. (2014). Transition to self-sufficient mixed crop-dairy farming systems. Renewable Agriculture and Food Systems, vol. 29, n 3, pp. 195-205.

Desriers M. (2011). Recensement agricole 2010. Structure des exploitations. Les productions se concentrent dans les exploitations spécialisées. Agreste Primeur, n 272, 4 p.

Devun J., Bébin D., Moreau S., Roulenc M., Manneville V., Chassaing C., Lherm M. (2014). Conséquences des pratiques fourragères et des caractéristiques du parcellaire sur la consommation de carburant dans les exploitations bovin viande du bassin Charolais. Rencontres Recherches Ruminants, $\mathrm{n}^{\circ} 21$, pp. 44.

Garambois N. (2011). Des prairies et des hommes : agro-écologie, création de richesse et emploi en élevage bovin. Paris, Thèse de doctorat, AgroParisTech, 595 p.

Garambois N., Devienne S. (2012). Les systèmes herbagers économes du Bocage 
Vendéen : une alternative pour un développement agricole durable ? Innovations Agronomiques, $\mathrm{n}^{\circ}$ 22, pp. 117-134.

Giroux G. (2011). Recensement agricole 2010. Premières tendances. Agreste Primeur, $\mathrm{n}^{\circ} 266,4 \mathrm{p}$.

Guihard V., Lesdos C. (2007). L'agriculture sur trente ans : une analyse comparative avec l'industrie et les services. In L'agriculture, nouveaux défis, Paris, INSEE, coll. «Références »,pp. 47-63.

Hallam . (1991). Economies of size and scale in agriculture: an interpretive review of empirical measurement. Review of Agricultural Economics, ${ }^{\circ} 13$, pp. 155-172.

Havet A., Coquil X., Fiorelli J. L., Gibon A., Martem G., Roche B., Ryschawy J., Schaller N., Dedieu B. (2014). Review of livestock farmer adaptation to increase forages in crop rotations in western France. Agriculture Ecosystems \& Environment, $\mathrm{n}^{\circ}$ 190, pp. 120-127.

Helfand S. M., Levine E. S. (2004). Farm size and the determinants of productive efficiency in the Brazilian Center-West. Agricultural Economics, $\mathrm{n}^{\circ} 31$, pp. 241-249.

Hostiou N., Dedieu B. (2011). A method for assessing work productivity and flexibility in livestock farms. Animal, n ${ }^{\circ}$ 6, pp. 852-862.

Hostiou N., Fagon J. (2012). Simplification des conduites d'élevage : analyse transversale des pratiques mises en œuvre dans les filières herbivores et granivores. INRA Productions Animales, ${ }^{\circ}$ 25, pp. 127-140.

Institut de l'Élevage (2011). L'élevage bovin allaitant français à l'horizon 2015 et perspectives 2035. Dossier Économie de l'Élevage, $\mathrm{n}^{\circ} 416,48 \mathrm{p}$.

Jaforullah M., Whiteman J. (1999). Scale efficiency in the New Zealand dairy industry: a non-parametric approach. The Australian Journal of Agricultural Ressource Economics, vol. 43, n 4, pp. 523-541.

Latruffe L. (2010). Competitiveness, productivity and Efficiency in the Agricultural and AgriFood Sectors. OECD Food, Agriculture and Fisheries Papers, $\mathrm{n}^{\circ} 30$, OECD Publishing. http://dx.doi.org/10.1787/5km91nkdt6d6-en

Lebacq T., Baret P. V., Stilman D. (2015). Role of input self-sufficiency in the economic and environmental sustainability of specialized dairy farms. Animal, n 9 , pp. 544-552.

Liénard G., Bébin D., Lherm M., Veysset P. (1998). Évolution des systèmes de récolte et d'élevage en exploitations herbagères de bovins allaitants. Cas du Charolais. Fourrages, $\mathrm{n}^{\circ} 155$, pp. 305-317.

Lherm M., Benoit M. (2003). L'autonomie de l'alimentation des systèmes d'élevage allaitant : évaluation et impacts économiques. Fourrages, $\mathrm{n}^{\circ}$ 176, pp. 411-424.

Madelrieux S., Dedieu B. (2008). Qualification and assessment of work organization in livestock farms. Animal, n 2, pp. 435-446.

Martel G., Dourmand J. Y., Dedieu B. (2008). Do labour productivity and preferences about work load distribution affect reproduction management and performance in pig farms? Livestock Science, $\mathrm{n}^{\circ} 118$, pp. 96-117.

Morrison Paul C., Nehring R., Banker D., Smwaru A. (2004). Scale economies and efficiency in U.S. agriculture: are traditional farms history? Journal of Productivity Analysis, $\mathrm{n}^{\circ}$ 22, pp. 185-205.

Mosheim R., Knox Lovell C. A. (2009). Scale economies and inefficiency of U.S. dairy farms. American Journal of Agricultural Economics, vol. 91, n 3, pp. 777-794.

Perrot C., Caillaud D., Chambaut H. (2013). Économies d'échelle et économies de gamme en production laitière. Analyse technico-économique et environnementale des exploitations de polyculture-élevage. Notes et études socio-économiques, $\mathrm{n}^{\circ} 37$, pp. 7-32.

Ripoll-Bosch R., Joy M., Bernués A. (2014). Role of self-sufficiency, productivity and diversification on the economic sustainability of farming systems with autochthonous sheep breeds in less favoured areas in Southern Europe. Animal, n 8, pp. 1229-1237.

Rouquette C., Baschet J. F. (2010). Le réseau d'information comptable agricole (RICA). MAAP, Service de la statistique et de la prospective, Centre d'Études et de Prospective, $\mathrm{n}^{\circ} 23,4 \mathrm{p}$.

Ryschawy J., Choisis N., Choisis J. P., Joannon A., Gibon A. (2012). Mixed crop-livestock systems: an economic and 
environmental-friendly way of farming? Animal, vol. 6, n 10, pp. 1722-1730.

Ryschawy J., Choisis N., Choisis J. P. Gibon A. (2013). Paths to last in mixed crop-livestock farming: lessons from an assessment of farm trajectories of change. Animal, vol. 7, n ${ }^{\circ}$, pp. 673-681.

Salton C. J., Mercante F. M., Tomazi M., Zanatta J. A., Concenço G., Silva W. M., Retore M. (2014). Integrated crop-livestock system in tropical Brazil: toward a sustainable production system. Agriculture Ecosystems \& Environment, $\mathrm{n}^{\circ} 190$, pp. 70-79.

Singbo A. G., Larue B. (2014). Scale economies and technical efficiency of Quebec dairy farms. CREATE, cahier de recherche/ Working Paper 2014-7, 42 p.

Sheng Y., Zhao S., Nossal K., Zhang D. (2014). Productivity and farm size in Australian agriculture: reinvesting the return to scale. Australian Journal of Agricultural and Resource Economics, n ${ }^{\circ}$ 59, pp. 16-38.

Thibert M., Berger J. (2009). Les prix agricoles, observation et mesure : l'indice des prix des produits agricoles à la production (IPPAP). Méthodologie de la base 2005. INSEE Méthodes, $\mathrm{n}^{\circ} 121,60 \mathrm{p}$.
Veysset P., Lherm M., Bébin D. (2004). Performances technico-économiques pour les grands troupeaux bovins allaitants : le cas du Charolais. Renc. Rech. Ruminants, $\mathrm{n}^{\circ}$ 11, pp. 141-144.

Veysset P., Lherm M., Bébin D. (2005). Évolutions, dispersions et déterminants du revenu en élevage bovin allaitant charolais. Étude sur 15 ans (1989-2003) à partir d'un échantillon constant de 69 exploitations. INRA Productions Animales, vol. 18, $\mathrm{n}^{\circ} 4$, pp. 265-275.

Veysset P., Bébin D., Lherm M. (2007). Impacts de la sécheresse de 2003 sur les résultats technico-économiques en élevage bovin allaitant Charolais. Fourrages, $\mathrm{n}^{\circ} 191$, pp. 311-322.

Veysset P., Benoit M., Laignel G., Bébin D., Roulenc M., Lherm M. (2014a). Analyse et déterminants de l'évolution des performances d'élevages bovins et ovins allaitants en zones défavorisées de 1990 à 2012. INRA Prod. Anim., vol. 27, $\mathrm{n}^{\circ}$ 1, pp. 49-64.

Veysset P., Lherm M., Bébin D., Roulenc M. (2014b). Mixed crop-livestock farming systems: a sustainable way to produce beef? Commercial farms results, questions and perspective. Animal, n 8, pp. 1218-1228. 